\title{
Análise e previsão da série recebimento e produção de leite da usina escola de laticinios da Universidade Federal de Santa Maria - RS
}

\author{
GiLvete Wolf Lírio \\ Danton Pierret \\ Vanusa Hackenhatr Pierret \\ AdRiano MEndonça Souza \\ Wesley Vieira da Silva
}

\section{Resumo}

Com a globalização o setor empresarial mundial está em uma fase de transição, buscando novas tecnologias com o objetivo de satisfazer um mercado cada vez mais exigente e informado. Nesse contexto e, à luz da Análise de Regressão Linear Dinâmica, procura-se avaliar a condução eficiente das ações gerenciais de planejamento, manutenção e melhoria da qualidade. Busca-se analisar a relação existente entre o recebimento do leite e a produção do leite pasteurizado, para fins de previsão em uma Usina de Laticínios de Santa Maria. Os dados coletados são diários e refere-se ao período compreendido entre maio e julho de 2002 da Usina Escola de Laticínios da Universidade Federal de Santa Maria - Santa Maria - RS, totalizando 83 observações. Com base nos resultados obtidos a partir das previsões, foi possível o melhor planejamento da produção da usina em função do volume de leite recebido, aceitando ou não determinados pedidos, além de traçar estratégias para driblar as dificuldades num curto espaço de tempo.

Palavras-Chave: Leite, Previsão, Planejamento no Curto Prazo e Análise de Regressão.. 


\section{Summary}

The world globalization bring to the market and to the business class a great phase of adaptation and search for new technologies to guaranty the quality of its products. The statistics is a useful tool to help to maintain the quality in a process or service. This way is possible to reduce costs and maintain the clients satisfied. The main purpose of this paper is to analyze the relationship between the milk received and the milk processed in the Usina de Laticínios de Santa Maria to forecast the next value. The data were daily collected since may/2002 till jul/2002 in a total of 83 observations, and were analyzed using the dynamic regression analysis. The results obtained was possible to forecast and better plan the production of milk received to be processed. That way the manager can accept or reject a new orders and can make a new strategies to avoid difficulties in a short term.

Key-words: Milk, Forecast, Planing in short time, Regression Analysis

\section{Introdução}

A crescente procura do consumidor por produtos e/ou serviços de qualidade têm exigido das indústrias de laticínios um maior empenho em relação aos padrões das matérias-primas recebidas, como também dos produtos industrializados ofertados no mercado. Além desta peculiaridade do mercado, foi somente a partir de 1996 que o governo federal, sob o pretexto de melhorar a qualidade do leite, começou a discutir uma nova regra para a produção de leite no Brasil, privilegiando o grande, em detrimento do pequeno produtor familiar. Essa exclusão de pequenos produtores de economia familiar começou a aumentar desde então.

Esta atividade é caracterizada como um negócio de lucros reduzidos, onde impõe que somente permanecerão no mercado aqueles que consigam reduzir os seus custos e aumentem o volume de leite a ser comercializado.

De acordo com a nova legislação para o leite (portaria $n^{\circ}$ 56 ), foram estabelecidas algumas normas para a produção de diversos tipos de leite no Brasil. Essas normas, que na maioria das vezes atingem aos pequenos produtores os quais, não possuem recursos 
para a sua implementação.

Em conseqüência disso, o número de pequenos produtores que fornecem leite vem diminuindo gradativamente. Um exemplo são os dados fornecidos pelo CNA/DECOM e EMBRAPA onde consta que em 1987, existiam 175.450 produtores de leite e em 2000 esse número caiu para 114.450 , ou seja, uma redução de $35 \%$. Em decorrência dessas novas exigências que assolam o pequeno produtor, grande parte deles reúne-se em pequenas cooperativas.

No caso específico deste trabalho, os dados foram coletados junto à Cooperativa dos Produtores de Leite de Santa Maria que é formada por 83 produtores, oriundos da região de Santa Maria, sendo que entregam a sua produção de leite para beneficiamento na Usina Escola de Laticínios da UFSM. Esta usina foi fundada na década de 1970 e hoje é explorada pela referida Cooperativa produzindo além do leite pasteurizado, queijo, iogurte, sorvete e nata.

Em função dos pedidos dos pontos de venda uma parte do recebimento do leite in natura é transformada em derivados e o restante é então pasteurizado para a comercialização como leite tipo C, sendo então, importante da produção desta matéria prima dado à possibilidade de planejar melhor o volume de leite a ser colocado no mercado consumidor.

Os dados da série trabalhada são diários e os valores referem-se ao volume de leite em litros, que são do período de maio a julho de 2002. No trabalho proposto será modelada uma equação de regressão linear dinâmica que procure relacionar a produção de leite pasteurizado e a variável recebimento do leite. O enfoque básico do trabalho será um estudo do comportamento das variáveis, com o objetivo principal de encontrar um modelo representativo que seja capaz de fazer uma previsão a curto prazo, utilizando-se a metodologia de análise de regressão dinâmica.

A implementação de previsões a curto prazo facilitará à gerência na tomada de decisões, onde será possível verificar a possibilidade de aceitar ou não os pedidos solicitados à Usina de Laticínios da UFSM, para a produção de outros itens, desta forma ocasionando uma adequação da demanda dos produtos derivados do leite, bem como o aumento da produção do leite pasteurizado. Finalmente, este trabalho encontra-se estruturado em quatro seções. A segunda seção mostra a metodologia a ser adotada, a terceira seção traz os resultados empíricos e a quarta seção enfoca as considerações finais e algumas recomendações. 


\section{Metodologia}

Este estudo procura analisar o recebimento diário de leite e a respectiva produção, pautando-se numa série temporal que cobre o período de três meses compreendidos entre maio e julho de 2002.

Inicialmente, fez-se a coleta dos dados, totalizando 92 observações de cada variável, onde foram retirados aqueles dados referentes aos domingos, pelo fato de ocorrer somente o recebimento do leite e não haver produção neste dia, trabalhando-se então, com 83 observações.

Num segundo momento, realizou-se o estudo do comportamento das respectivas variáveis, através de estatísticas descritivas com vistas a verificar o comportamento das variáveis.

Posteriormente, utilizou-se a metodologia para a modelagem das variáveis, valendo-se da metodologia da escola de Londres "do geral para específico", onde atribui-se um elevado número de defasagens sendo paulatinamente eliminadas aquelas variáveis menos significativas estatisticamente, com vistas a encontrar um modelo parcimonioso e capaz de realizar boas previsões.

\subsection{Modelo de Regressão Dinâmica}

Segundo Vasconcelos e Alves (2000), um modelo nada mais é do que uma representação simplificada de um processo real. Por isso, deve ser simples o bastante para demonstrar o comportamento das variáveis em estudo e, a partir deste modelo pode-se projetar hipóteses que retratem de forma clara e precisa a realidade dos dados para a futura tomada de decisão. Um instrumento bastante eficaz para a construção de um modelo é a análise de regressão, dado que ela é utilizada principalmente com o objetivo de realizar previsões.

A análise de regressão compreende quatro etapas, que são: especificação do modelo, estimação do modelo, análise dos resultados e previsão. Para iniciar o estudo mais acurado das variáveis do modelo, é necessário verificar a priori, qual variável será a dependente ou explicada e quais serão as variáveis independentes ou explicativas.

No caso específico deste estudo, a variável dependente 
será a produção de leite pasteurizado, enquanto a variável independente será o recebimento de leite. Chamar-se-á a partir de agora $Y$ a variável produção de leite e $\mathrm{X}$ a variável recebimento de leite.

No modelo que será demonstrado neste trabalho, utilizarse-á o modelo de regressão linear dinâmica, o qual leva em consideração os efeitos ocorridos em períodos de tempo anteriores (defasagens). Supondo que a forma seja linear, o modelo de defasagem ou modelo dinâmico para $t=n+1, \ldots T$, pode ser visualizado como segue:

$$
Y_{t}=\alpha+\beta_{0} x_{t}+\beta_{1} x_{t-1}+\cdots+\beta_{n} x_{t-n}+e_{t}
$$

Observe através da expressão (1) que, obtendo-se T observações sobre os pares $\left(x_{t}, y_{t}\right)$, então só se dispõe de $T-n$ observações completas para estimação, pois $n$ observações se perdem ao criar $x_{t-1}, x_{t-2}, \ldots x_{t-n}$. Veja que na defasagem da expressão (1), o parâmetro a é o intercepto, enquanto que o parâmetro b é chamado peso da defasagem distribuída.

Se ao longo do tempo xt segue um padrão, então $x_{t-1}$ seguirá padrão análogo fazendo com que $x_{t}$ e $x_{t-1}$ sejam correlacionados (Hill, Griffiths e Judge, 1999).

\subsubsection{As Defasagens Aritméticas}

Os pesos das defasagens diminuem linearmente e para tanto, impôs aos pesos de defasagem distribuídas restrições da forma:

$$
\begin{aligned}
& \beta_{0}=(n+1) \gamma \\
& \beta_{1}=n \gamma \\
& \beta_{2}=(n-1) \gamma \\
& \beta_{n}=\gamma
\end{aligned}
$$

onde, $\gamma>0$ é uma constante positiva.

A expressão (2) pode ser levada ao modelo de defasagem finita em (1) e simplificada, para estimar a defasagem aritmética utilizando-se, por exemplo, uma defasagem de $n=4$ períodos, sendo $t=5, \ldots, T$. Logo, o modelo de defasagem finita pode ser escrito da 
seguinte forma:

$$
Y_{t}=\alpha+\beta_{0} X_{t}+\beta_{1} X_{t-1}+\beta_{2} X_{t-2}+\beta_{3} X_{t-3}+\beta_{4} X_{t-4}+\ell_{t}
$$

Neste estudo a modelagem obedece à metodologia do geral para o específico "the General to Specific" da Escola de Economia de Londres (London School of Economics). Para o caso das estimações econométricas realizadas através do Método dos Mínimos Quadrados Ordinários (OLS), usa-se o mesmo método desenvolvido por Davidson et al. (1978, p. 661-692), onde eles procuram modelar uma função consumo para o Reino Unido. Neste caso, parte-se de um modelo matemático especificado, onde se leva em conta uma elevada estrutura de defasagens em todas as variáveis do modelo.

Nesse prisma, a avaliação diagnóstico dessas regressões pode ser feita considerando a imposição de algumas restrições lineares no modelo e, gradativamente, à luz dos testes t-Student e FSnedecor determina-se um modelo simples (parcimonioso) e bem especificado a ser usado para fins de previsão.

Para Makridakis et al. (1998), essa metodologia de previsão baseia suas previsões na extrapolação de características de observações passadas e no inter-relacionamento entre tais observações, fornecendo previsões acuradas, se o futuro apresentar um comportamento similar ao passado.

Os autores reportados anteriormente ainda salientam que, apesar de boa parte dos métodos de previsão de séries temporais estarem calcados nas análises de observações das séries de interesse, para a especificação de algum modelo que descreva tais observações, alguns procedimentos de previsão procuram explicar o comportamento de uma série temporal pela evolução dos fenômenos observacionais de outras séries. Notadamente, esses modelos devem garantir todas as hipóteses de normalidade, inferidas sobre o vetor de resíduos provenientes das estimações.

Alguns testes bastante comuns nessa mesma metodologia econométrica devem ser levados em consideração. O teste de Autocorrelação Serial, conhecido como Durbin-Watson (DW) por exemplo, procura testar a hipótese nula de não autocorrelação serial ao nível de significância de $5 \%$ e 10\%. Por outro lado, o teste "ARCH" procura testar a presença ou não de resíduos autocorrelacionados heteroscedásticos, bem como o teste " $\mathrm{X}^{211}$ que visa avaliar a presen- 
ça ou não de heteroscedasticidade dos resíduos avaliando se os resíduos forem provenientes do modelo homoscedástico ao nível de significância de $5 \%$.

Já o teste de normalidade, conhecido como Jarque-Bera, segue uma distribuição do tipo qui-quadrado com dois graus de liberdade. O seu valor crítico ao nível de significância de $5 \%$ é igual a 5,99 , para conduzir à aceitação da hipótese nula de que a distribuição dos resíduos do modelo estimado é gaussiana.

O Método de estimação dos parâmetros do modelo é o de Mínimos Quadrados Ordinários (MQO), o qual possui algumas propriedades estatísticas interessantes, que o fizeram um dos métodos mais eficientes e usados na análise de regressão (Gujarati, 2000).

O método dos mínimos quadrados permite então, determinar a reta que passa com melhor ajustamento entre os pontos do diagrama, de forma a selecionar b1 e b2 tais que, a soma dos quadrados dos resíduos seja mínima.

\section{Resultados empíricos}

Procurou-se analisar os dados através de um estudo das variáveis: recebimento e produção de leite e a análise descritiva das variáveis, está representada na tabela 01 a seguir:

Tab. 1. Valores das estatísticas descritivas para as variáveis: recebimento de leite e produção de leite

\begin{tabular}{cccc}
\hline VARIÁVEIS & MÉDIA & DESVIO-PADRÃO & COEF. VARIAÇÃo \\
\hline Recebimento Leite & 2931,3132 & 557,2867836 & 19,011506 \\
Produção Leite & 2500,096 & 629,4257 & 25,176061 \\
\hline
\end{tabular}

Observando-se a Tabela 01, verificou-se que as variáveis apresentam uma baixa oscilação em torno da média, sendo esta representativa. Esta baixa variabilidade certamente acarretará parâmetros que apresentam uma constância ao longo do tempo. Por 
outro lado, na figura 1, é fácil verificar que as séries temporais de recebimento e produção de leite aparentam ser estacionárias.

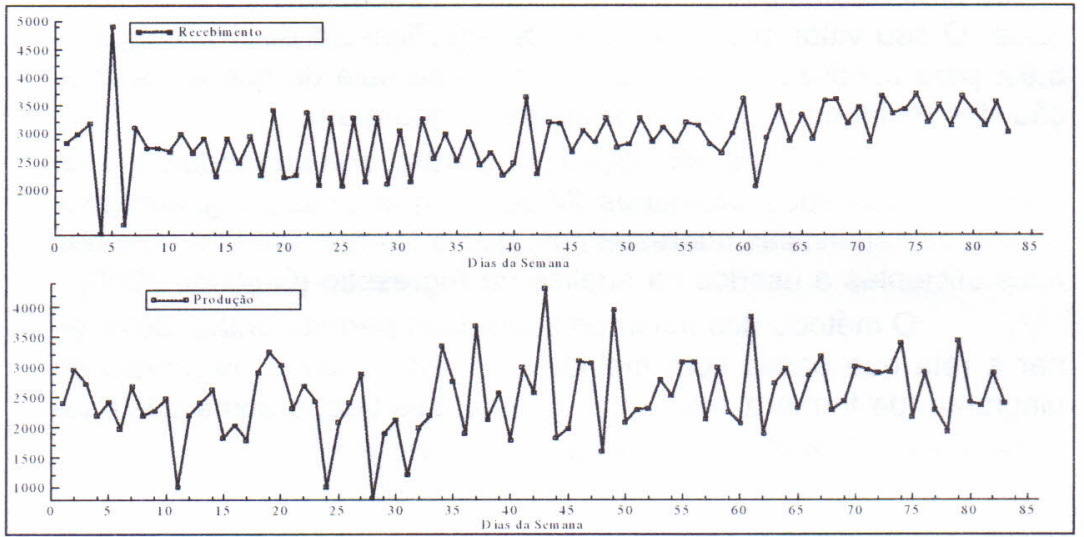

Fig. 1. Comportamento das Séries de Recebimento e Produção de leite

Observa-se ainda que as respectivas séries seguem o mesmo comportamento temporal que situa-se em torno da média, com variabilidade muito próximas, mesmo apresentando alguns pontos discrepantes ao longo das unidades amostrais.

Os testes utilizados com maior freqüência para verificação se existem ou não raízes unitárias ${ }^{1}$ são baseados nas estatísticas de Dickey-Fuller (DF) ${ }^{2}$ e Dickey-Fuller Aumentado (ADF) ${ }^{3}$. O conceito de co-integração foi preconizado por Granger (1981) e posteriormente ampliado por Engle e Ganger (1987) ${ }^{4}$, os quais consideram

\footnotetext{
'As metodologias pormenorizadas sobre esses testes e aplicações são discutidas em Fuller (1976), Dickey, Fuller (1981) e (1994).

${ }^{2} A$ estatística $D F$ é empregada para testar se um processo $A R(1)$ tem uma raiz unitária.

${ }^{3} A$ estatística aplica-se ao caso em que $y$ é gerado por um pro-cesso $A R(p), p>1$. ${ }^{4}$ Maiores detalhes sobre co-integração e seus procedimentos podem ser obtidos em Engle, Yoo Engle,
} 
que uma série temporal $x_{t}$, sem tendência determinística, é integrada de ordem $p, I(p)$, se esta possuir uma representação estacionária e inversível do tipo Auto Regressivo de Médias Móveis (ARMA) em razão de ter sido diferenciada $p$ vezes.

Os métodos clássicos de estimação econométrica tomam como pressuposto o fato de a média e a variância das variáveis serem constantes e independentes do tempo. Contudo, a aplicação dos testes de raiz unitária tem mostrado que esses pressupostos nem sempre são válidos para um grande número de variáveis macroeconômicas de séries temporais. Ele enfatiza que as variáveis, cujas médias e variâncias modificam-se ao longo do tempo, são denominadas de não estacionárias ou variáveis com a presença de raiz unitária.

Cabe salientar que, todos os dados referem-se a valores nominais no período considerado. Eles foram codificados, visando facilitar o seu manuseio algébrico. Por outro lado, os softwares utilizados nas estimações econométricas foram o PcGive 9.0 e o EViews 3.0, valendo-se de uma estrutura dinâmica que fora mencionada anteriormente. $\mathrm{Na}$ tabela a seguir evidencia-se o teste de raiz unitária conhecido com Dickey-Fuller (DF) e Dickey-Fuller Ampliado (ADF) para as respectivas variáveis.

Os testes foram realizados em três versões: sem constante, com constante e com constante e tendência. Partiu-se inicialmente de um número de cinco defasagens para cada variável, sendo feitas às devidas reduções, escolhendo-se aquela defasagem que minimiza o critério bayesiano de Schwarz (SC).

Tab. 2. Resultados dos Testes de Raiz Unitária

\begin{tabular}{ccccc}
\hline Variáveis & $\begin{array}{c}t \text {-ADF: com } \\
\text { constante }\end{array}$ & $\begin{array}{c}\mathrm{N}^{\circ} \text { de } \\
\text { defasagens } \\
\text { com maior t-ADF }\end{array}$ & $\begin{array}{c}t \text {-ADF: com } \\
\text { constante e } \\
\text { Tendência }\end{array}$ & $\begin{array}{c}\mathrm{N}^{\circ} \text { de } \\
\text { defasagens } \\
\text { com maior } t \text {-ADF }\end{array}$ \\
\hline Recebimento & $-3,8295^{(*)}$ & 1 & $-3,5307^{(*)}$ & 3 \\
Produção & $-4,1158^{(*)}$ & 2 & $-4,0617^{(*)}$ & 4 \\
\hline
\end{tabular}

Nota: Os valores críticos para o teste ADF com constante e tendência foram iguais a $-3,468$ para $5 \%$ e $-4,080$ para $1 \%$. Já os valores críticos para o mesmo teste sem a tendência foram iguais a $-2,889$ para $5 \%$ e $-3,516$ para $1 \%$. 
Vale salientar que os valores críticos baseiam-se em Mackinnon (1991). Logo, de acordo com a tabela 02, pode-se inferir que todas as variáveis estudadas são estacionárias de primeira ordem aos niveis de significância estatística de 5\% e 1\%, considerando um modelo com e sem a tendência determinística. Os resultados mostram a rejeição da hipótese nula de raiz unitária nas variáveis referenciadas anteriormente.

A partir dos resultados obtidos na tabela 02, busca-se então as estimações dos parâmetros do modelo. O modelo a ser estimado parte de uma estrutura de 5 defasagens em cada uma das variáveis. Considerou-se que essa estrutura de defasagens corresponde ao período de funcionamento regular em dias úteis na fábrica.

Os parâmetros foram estimados em sua forma logarítmica, uma vez que esse tipo de transformação matemática possui a vantagem de se obter as elasticidades, o que facilita de sobremaneira as interpretações dos coeficientes em termos econômicos. Os valores estimados para os coeficientes do modelo formulado encontram-se na tabela a seguir.

Tab. 3. Estimação dos Parâmetros do Modelo Econométrico

\begin{tabular}{lllll}
\hline Variáveis & Parâmetros & Erro-Padrão & $\boldsymbol{t}$-Student & Significância \\
\hline Ln (Rt-1) & 0,5294 & 0,08994 & 5,8866 & 0,0000 \\
Ln (Rt-2) & 0,4484 & 0,08997 & 4,9839 & 0,0000 \\
& & & & \\
R2 $=6,38 \% ;$ & F+ 5,3872 com: $P=0,0229 ;$ & $D W=2,16 ;$ & $R S S=6,1695$ \\
\hline
\end{tabular}

Observe na tabela 03, que os únicos parâmetros estatisticamente significativos foram o logaritmo neperiano dos recebimentos de leite nos períodos ( $\mathrm{t}-1)$ e ( $\mathrm{t}-2)$, com coeficientes onde o teste tstudent foram superiores ao valor tabelado que é igual a 2. Logo, o modelo estimado configura-se num autoregressivo de segunda ordem, já que os demais parâmetros foram retirados paulatinamente durante o processo de estimação, à medida que o respectivo teste 
apresentasse valores abaixo daquele tabelado.

Por outro lado, o modelo estimado apresentou uma estatística Durbin-Watson (DW) muito próximo de 2, que é o valor considerado ideal para se aceitar a hipótese nula de não autocorrelação. Apesar do modelo possuir um baixo poder explicativo em termos do coeficiente de explicação ele também evidenciou que os parâmetros que foram determinados são estáveis ao longo do tempo, não apresentando qualquer tipo de quebra estrutural no teste de soma acumuladas dos resíduos oriundos do modelo (figura 2.a). Além disso, o teste de Chow para os resíduos recursivos (figura 2.b) evidencia 3 quebras estruturais nos pontos 11, 24 e 28, que não afetaram a capacidade preditiva do modelo. Os gráficos mencionados podem ser vistos na figura 02 a seguir.

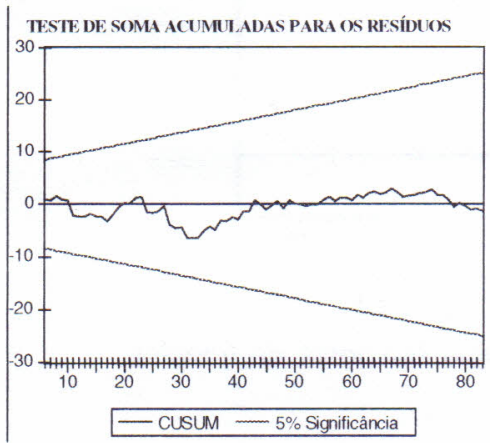

(a)
TESTE DE CHOW PARA O COMPORTAMENTO DOS RESÍDUOS RECURSIVOS

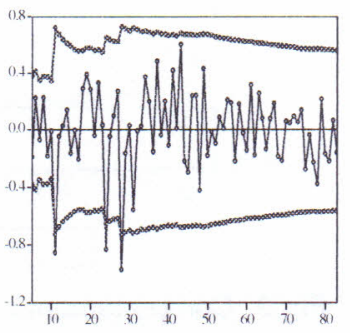

(b)

Fig. 2. Testes de Soma Acumuladas para os resíduos e de Chow para os Resíduos Recursivos para o Modelo Formulado

Uma vez que os coeficientes estimados são as elasticidades ou coeficientes de sensibilidades, estes mostraram ser estáveis ao longo do tempo, com valores abaixo da unidade. Assim, tais coeficientes podem ser interpretados tomando-se por base o conceito tradicional elasticidade encontrados nos manuais de microeconomia. Logo, percebe-se que elevações em torno de $10 \%$ no recebimento do produto in natura fazem com que haja um aumento de cerca de $5,3 \%$ na produção num instante (t- 1 ) e de $4,48 \%$ no segundo instante $(\mathrm{t}-2)$, tudo mais é mantido constante. 
Por outro lado, avaliou-se ainda a função resposta a impulsos na equação de estabilidade que foi formulada considerando a resposta dada pela produção de leite, em função de um choque temporário nos recebimentos da matéria-prima ou seja, dado um choque de uma unidade de desvio-padrão nos recebimentos de leite, como se comportará a produção de leite pasteurizado para fins de ajustamento do modelo. A figura a seguir evidencia a função resposta a impulsos.

Resposta da Produção de Leite a um Impulso de uma Unidade de Desvio-padrão nos Recebimentos

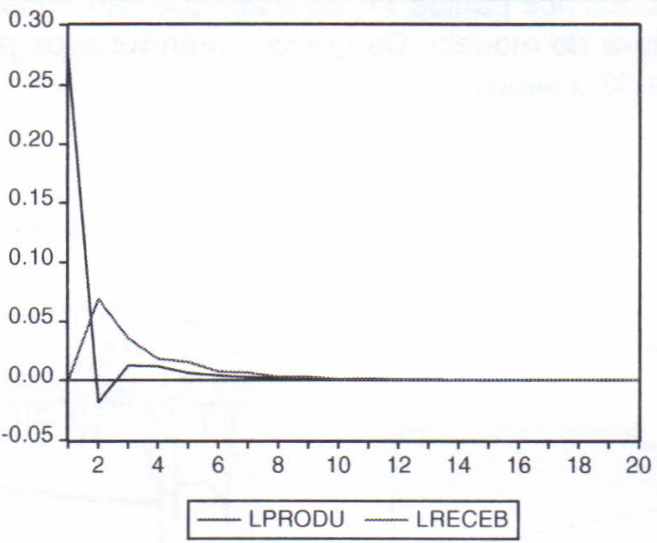

Fig.3. Comportamento da Função Resposta de Impulso

Observe através da figura 3 que um choque temporário na variável recebimento do leite in natura faz com que a produção de leite pasteurizado se ajuste após o segundo dia, não havendo a partir daí, perda de fornecimento por parte do consumidor final, o que pode ser considerado um resultado bastante aceitável a essa absorção do choque.

Testou-se também, o modelo formulado em termos de previsões. Inicialmente estabeleceu-se previsões ex-post (dentro da amostra) e logo em seguida, as previsões ex-ante (fora da amostra) considerando 3 cenários alternativos. O primeiro cenário considerado pessimista, onde supõe-se que haja uma queda de $10 \%$ no recebimento do leite in natura, o segundo cenário será o conservador, 
onde o recebimento irá crescer a mesma taxa durante todo o período amostrado e, por último, o terceiro cenário será o otimista, onde o fornecimento de leite aumentará para a Usina de Laticínios da UFSM em torno de $10 \%$, tudo o mais mantido constante. Os valores previstos dentro da amostra encontram-se na tabela 4 .

Tab. 4. Previsões Ex-Post da Produção de Leite

\begin{tabular}{ccccc}
\hline Observações & Produção & Produção(Prevista) & $\begin{array}{c}\text { Residuos } \\
(y-y)\end{array}$ & t-Student \\
\hline 79 & 3447 & 26467 & 800 & 0,909945 \\
80 & 2420 & 2719 & -299 & $-0,399555$ \\
81 & 2370 & 2760 & -390 & $-0,519691$ \\
82 & 2910 & 2620 & 290 & 0,362266 \\
83 & 2400 & 2688 & -288 & $-0,390261$ \\
\hline
\end{tabular}

Observe ainda que todos os valores expressos em sua forma logarítmica, encontram-se nos intervalos de mais ou menos dois desvios-padrão mostrado na figura 04 e percebe-se que a constância dos parâmetros significa a garantia de qualidade na prestação de serviços e produtos derivados do leite, bem como o leite pasteurizado, isso corrobora com a boa performance do modelo estimado.

No que concerne às previsões ex-ante, percebe-se à luz da tabela 05 , os três cenários levados em consideração. O primeiro cenário evidencia queda de $10 \%$ no fornecimento do leite in natura, admitindo por hipótese que haverá uma piora nas condições de tratamento do rebanho leiteiro da região, além de problemas sazonais que vem se tornando comuns na região, isso proporciona quedas na produção de leite pasteurizado, tal como visualiza-se na segunda coluna da tabela a seguir.

Por outro lado, no segundo cenário a produção de leite crescerá à taxa média de $8,26 \%$. Essa taxa foi obtida mediante a extração da média aritmética simples da taxa de crescimento da produção de leite no período analisado. Finalmente, a tabela 05 aponta 
na quarta coluna, o cenário otimista, considerou-se um crescimento da produção de leite pasteurizado em torno de 10\% admitindo-se, por suposição, uma melhoria significativa das condições de criação e produtividade do rebanho leiteiro na região, além de uma contribuição dos fatores sazonais.

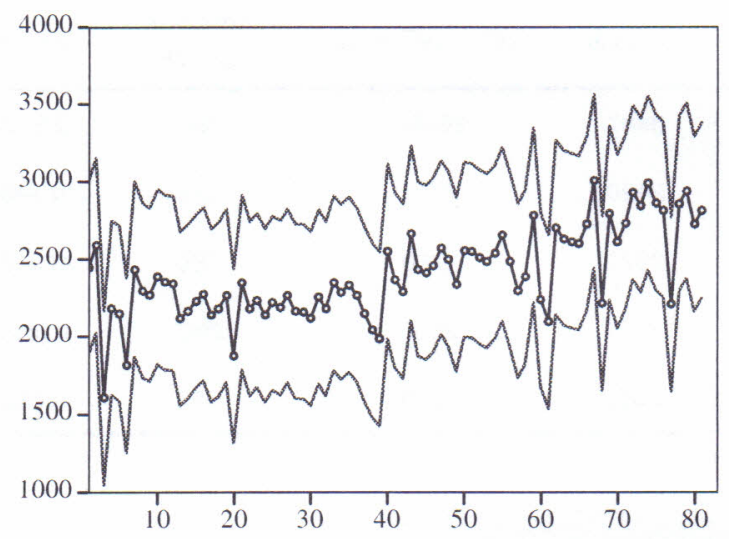

Fig. 4. Previsão da Produção de Leite da Usina da UFSM

Tab. 5. Cenários das Previsões Ex-Ante da Produção de Leite

\begin{tabular}{cccc}
\hline Previsões & Cenário Pessimista & Cenário Conservador & Cenário Otimista \\
\hline 84 & 2531 & 3045 & 3094 \\
85 & 2278 & 3296 & 3403 \\
86 & 2050 & 3568 & 3743 \\
87 & 1845 & 3863 & 4117 \\
88 & 1661 & 4182 & 4529 \\
\hline
\end{tabular}


Finalmente, através da previsão da oferta desse produto no mercado consumidor o planejador poderá rejeitar ou não pedidos a priori, em função do volume recebido de leite in natura na usina, de tal modo que esse produto perecivel não fique estocado em locais onde os custos operacionais venham onerar de forma significativa as receitas dos cooperados.

\section{Considerações finais}

Este trabalho teve o propósito de estabelecer um modelo de previsão para a produção de leite pasteurizado com base nos recebimentos de leite in natura da Usina Escola de Laticínios da Universidade Federal de Santa Maria - Santa Maria - RS, baseandose em dados diários que cobrem o período de maio a julho de 2002 , totalizando 83 observações.

A metodologia de estimação utilizada foi o método dos mínimos quadrados ordinários (OLS), aliado à análise de cointegração numa abordagem "the general to specific" da escola de economia de Londres (London School of Economics).

As estimativas efetuadas para os valores em logaritmo do recebimento de leite in natura e a produção do leite pasteurizado mostraram-se satisfatórias no que diz respeito aos testes estatístico diagnóstico que foram utilizados para avaliar o modelo formulado.

Partiu-se de uma estrutura de 5 defasagens em cada uma das variáveis consideradas, sendo gradativamente eliminadas em função de não apresentarem coeficientes t-Student maiores ou iguais a 2. Encontrou-se com isso, um modelo parcimonioso onde a produção de leite pode ser prevista pela variável recebimento de leite no primeiro e segundo períodos imediatamente anteriores.

Os valores dos coeficientes que foram estimados são as elasticidades e mostrou-se ser inelástico, já que situou-se abaixo da unidade. Por outro lado, os valores previstos dentro da amostra mostraram-se estáveis, uma vez que forneceram previsões dentro da amostra acuradas estatisticamente, encontrando-se no intervalo de previsão de dois desvios-padrão.

No que tange as previsões fora da amostra, estabeleceuse alguns cenários alternativos, partindo-se de algumas suposições. No primeiro cenário levou-se em conta uma queda de $10 \%$ na produção do leite pasteurizado (cenário conservador). No segundo cenário a produção permaneceu com a mesma taxa de crescimento, que ficou em torno de $8,26 \%$ e, por último, o terceiro cenário foi o otimis- 
ta que levou-se em conta a suposição de uma taxa de crescimento de $10 \%$, supondo uma melhoria na infra-estrutura da criação do gado leiteiro.

Baseados nestas previsões a gerência da Usina de Laticínios é capaz de fazer uma programação de produção interna, dando preferência a determinados produtos como iogurte, sorvete, nata entre outros. Esta previsão faz-se necessária pois nos períodos de entre safra o volume de leite entregue pelos fornecedores não é constante, tendo neste período a necessidade de utilizar o número maior de fornecedores para que a usina não diminua sua produção de leite e seus derivados, mantendo desta maneira o nível de produção dos derivados do leite.

Finalmente, pode-se inferir à luz do modelo que fora estimado através de técnicas econométricas que ele foi considerado adequado, para se realizar a previsão da variável produção de leite pasteurizado dado a não violação das hipóteses clássicas dos modelos de regressão.

\section{Agradecimentos}

Agradecemos a Usina Escola de Laticínios de Santa Maria que nos forneceu os dados e também a Cooperativa de Produtores de leite de Santa Maria, que é formada por 83 produtores da região, sem a qual o nosso trabalho não teria sido possível e aos revisores pela sua contribuição. 


\section{Bibliografia}

BACCHI, Miriam. R. P. Previsão de preços de bovino, suíno e frango com modelos de séries temporais. Tese de Doutorado. Piracicaba: ESALQ, 1994. 173p.

DAVIDSON, J. H., D. H., HENDRY, F. Srba e . Yco. Econometric Modelling of the Aggregate Time-Series Relationship Between Consumers Expenditure and Income in the United Kingdom. The Economic Journal. v. 88, p. 661-692, 1978.

DICKEY, David A., FULLER, Wayne A. Likelihood ratio statistcs for autoregressive time series with a unit root. Econométrica, v.49, n.4, p.1057-72, July 1981.

ENGLE, Robert F. e GRANGER, C. W. J. Cointegration and error correction: Representation, estimation and testing. Econometric, 55(2):251-76 1987.

YOO, Byung S. Forecasting and testing in co-integrateal systems. Journal of Econometrics, North-Holland, v.35, n.1, p.14359, 1987.

ENGLE, Robert F.; GRANGER, C. W. J. Long-run economic relationship: readings in cointegration. New York: Oxford University Press, 1991. 301p. (Advanced texts in econometrics).

FERREIRA, Afonso H. B. Testes de cointegração e um modelo de correção de erro para a balança comercial brasileira. Estudos Econốmicos, São Paulo, v.23, n.1, p.35-65, jan./abr. 1993.

FULLER, Wayne A. Introduction to statistical time series. New York: John Wiley, 1976. 352p.

GRANGER, Clive W. J. Some properties of time series data and their use in econometric model specification. Jour-nal of Econometrics, North-Holland, v.16, n.1, p.121-30, May 1981.

GUJARATI, D. N. Econometria Básica. São Paulo: Makron Books, 2000.

HARRIS, Richard I.D. Cointegration analysis in econometric modelling. London: Prentice Hall, 1995. 176p

HILL, R. Carter, GRIFFITHS, W.E., JUDGE, G.G. Econometria. São Paulo: Saraiva, 1999.

MACKINNON, James G. Critical values for cointegration tests. In: ENGLE, Robert F.; GRANGER, W. J. Long-run economic relationships: readings in cointegration. New York: Oxford University Press, 1991. p.267-76.

MAKRIDAKIS, S., WHEELWRIGHT, S. C. e HYNDMAN, R. J. Forecasting: methods and applications. John Wiley \& Sons, Inc., 1998.

VASCONCELOS, M. A. S. e Alves, D. Manual de Econometria. São Paulo: Atlas, 2000. 308 p. 
Gilvete Wolf LíRIO ajlirio@ terra.com.br

Danton Pierret, Vanusa Hackenhaar Pierret PPGEP - UFSM - Santa Maria, RS - Brasil Adriano Mendonça Souza amsouza@smail.ufsm.br Departamento de Estatística CCNE - UFSM - Santa Maria, RS - Brasil Wesley Vieira da Silva Faculdades Integradas ASSESC - Florianópolis, SC - Brasil 